\title{
Fluid Structure Interaction Simulation of Left Ventricular Flow Dynamics under Left Ventricular Assist Device Support
}

\author{
C. W. Ong, B. T. Chan, E. Lim, N. A. Abu Osman, A. A. Abed, S. Dokos, N. H. Lovell
}

\begin{abstract}
For patient's receiving mechanical circulatory support, malfunction of the left ventricular assist device (LVADs) as well as mal-positioning of the cannula imposes serious threats to their life. It is therefore important to characterize the flow pattern and pressure distribution within the ventricle in the presence of an LVAD. In this paper, we present a $2 \mathrm{D}$ axisymmetric fluid structure interaction model of the passive left ventricle (LV) incorporating an LVAD cannula to simulate the effect of the LVAD cannula placement on the vortex dynamics. Results showed that larger recirculation area was formed at the cannula tip with increasing cannula insertion depth, and this is believed to reduce the risk of thrombus formation. Furthermore, we also simulated suction events (collapse of the LV) by closing the inlet. Vortex patterns were significantly altered under this condition, and the greatest LV wall displacement was observed at the part of the myocardium closest to the cannula tip.
\end{abstract}

Keywords: LVAD, heart pump, cannula, suction, vortex

\section{INTRODUCTION}

Congestive heart failure is a serious health condition characterized by the inability of the heart to supply adequate blood flow and therefore oxygen delivery to the body. Due to limited availability of donor organs and limitations in drug therapies, many left ventricular assist devices (LVADs) have been developed. In order to optimize LVAD use in unloading the ventricle, a detailed understanding of the impact of the device on the hemodynamic variables in the ventricles, such as flow dynamics and pressure, as well as cardiac wall motion, is crucial [1]. This can be achieved through in vivo animal experiments, clinical trials, in vitro experiments and numerical models.

Among these methods, computational fluid dynamics (CFD), which provides detailed flow characteristics in the ventricle, such as velocity profiles, shear rate, recirculation and pressure, have been widely used in the development of VADs [2]. CFD has been extensively used in the analysis and design of such LVADs, for example to determine the pressure-flow characteristics and efficiencies of the pump $[3,4]$. It has also been used to evaluate the thrombosis potential in the LVAD cannula with various geometrical designs [5]. In

C.W. Ong, B.T. Chan, E.Lim, N.A.Abu Osman are with the Department of Biomedical Engineering, Faculty of Engineering, University of Malaya, Kuala Lumpur 50603, Malaysia. phone: 603-7967 7022; fax: 603-7956 0027; e-mail: einly_lim@um.edu.my

A. A. Abed, S Dokos and N H Lovell are with Graduate School of Biomedical Engineering, University of New South Wales, Sydney 2052, Australia. email: s.dokos.@unsw.edu.au another study, Loerakker et al. [6] developed an axisymmetric fluid dynamic model of the left ventricle (LV), coupled to a lumped parameter model of the complete circulation, to investigate the influence of a LVAD on the vortex dynamics in a ventricle with dilated cardiomyopathy.

One limitation of the previous studies is that it does not take into account the interaction between the blood, the myocardium wall and the cannula, which are crucial to fully capture the impact of LVADs on the ventricular function [1]. Fluid structure interaction (FSI) models take into consideration the myocardium properties and provide important information such as myocardial displacement and wall stress. Although numerous FSI models for the ventricles have been published [7-9], they have not yet been used in the study of LVAD-ventricle interaction. So far, we are only aware of one study by McCormick et al. [1] which developed a fluid-solid coupled model incorporating fictitious-domain method to simulate the contact of the ventricular wall with the cannula placed at the apex.

In the present paper, we present an axisymmetric model of the passive $\mathrm{LV}$, incorporating an inflow cannula, to simulate the effect of LVAD cannula placement on the vortex dynamics, taking into account the coupling between the blood and the myocardial wall. Using the same model, we simulated suction events by closing the inlet. The two studies demonstrate the capability of the model to simulate the large deformations experienced by the ventricular wall under LVAD support.

\section{METHODS AND MATERIAL}

\section{A. Model description}

The present model consists of two solid materials, i.e. the myocardial wall and the cannula, as well as the fluid representing the blood in the LV (Fig. 1).

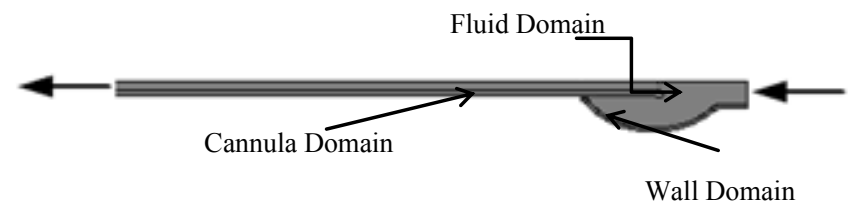

Fig. 1:.Diagram showing the fluid domain, wall domain and cannura domain in axisymmetric form when the cannula is inserted half way into ventricle. The arrow direction shows the blood flow across the cavity during filling phase.

The LV is modeled using a prolate ellipsoid with a long axis of $8 \mathrm{~cm}$ and a short axis of $5 \mathrm{~cm}$. A $1 \mathrm{~cm}$ tube with a diameter 
of $2.5 \mathrm{~cm}$ is connected to the upper end of the ellipse to represent the inflow passage. A thin-walled silicone cannula (Young's Modulus $=170 \mathrm{GPa}$ ) with a diameter of $1 \mathrm{~cm}$ and a length of $30 \mathrm{~cm}$ is inserted into the ventricle. A Mooney-Rivlin hyperelastic wall with a thickness of $0.2 \mathrm{~cm}$ is added outside the ventricular cavity. The strain energy density function, $\mathrm{W}_{\mathrm{s}}$, is adapted from [10],

$$
W_{S}=C_{10}\left(I_{1}-3\right)+C_{01}\left(I_{2}-3\right)+\frac{1}{2} k\left(J_{e l}-1\right)^{2}
$$

where $I_{1}$ and $I_{2}$ are the first and second invariant of the left isochoric Cauchy-Green deformation tensor, $J_{\text {el }}$ is the elastic Jacobian, $C_{i, j}$ are coefficients in the polynomial, and $k$ is the bulk modulus; $\mathrm{C}_{10}=10 \mathrm{kPa}, \mathrm{C}_{01}=2 \mathrm{kPa}, \rho=1000 \mathrm{~kg} / \mathrm{m}^{3}$, and $k=100 \mathrm{kPa}$. The incompressible fluid inside the cavity has a dynamic viscosity of $0.00316 \mathrm{~Pa} \cdot \mathrm{s}$ and a density of $1050 \mathrm{~kg} / \mathrm{m}$ [9].

\section{B. Numerical scheme}

In the present study, the FSI module provided by the commercial software, COMSOL was chosen to solve the model. The FSI interface uses an arbitrary Lagrangian-Eulerian (ALE) method to combine the fluid flow formulated using an Eulerian description with solid mechanics formulated using a Lagrangian description.

The fluid flow in the ventricle is described by the incompressible Navier Stokes equation (2) and the mass continuity equation (3).

$$
\begin{gathered}
\rho\left(\frac{\partial u}{\partial t}+\left(u_{f}-u_{m}\right)-\nabla u_{f}\right)=\nabla \cdot\left[-p \mathrm{I}+\mu\left(\nabla u_{f}+\left(\nabla u_{f}\right)^{T}\right)\right] \\
\rho \nabla \cdot u_{f}=0
\end{gathered}
$$

where $\rho$ is the fluid density, $u_{f}$ is the velocity vector of the fluid in a fixed coordinate system, $\boldsymbol{u}_{m}$ is the velocity vector of the fluid in a moving coordinate system, $p$ is the pressure and $\mathrm{I}$ is the unit tensor.

Once the above equations are solved using weak form formulation, load from the fluid $(\tau)$, i.e. the summation of pressure and viscous forces, can be calculated using

$$
\tau=-p \mathrm{I}+\mu\left(\nabla \mathrm{u}_{\mathrm{f}}+\left(\nabla u_{f}\right)^{T}\right)
$$

Load from the fluid is applied on the solid at the fluid-solid interface boundary, i.e.

$$
\tau \cdot n=\sigma \cdot n
$$

where $\sigma$ is the stress imposed on and $n$ stands for the interface normal.

The calculated solid stress is then used to solve for solid displacement, $u_{\text {solid }}$

$$
\rho \frac{\partial^{2} u_{\text {solid }}}{\partial t^{2}}-\nabla \cdot \sigma=0
$$

The resultant solid displacement is used to calculate the fluid velocity, given that at the fluid-solid interface, the velocity of the fluid is equal to the velocity of the wall $u_{w}$

$$
u_{\text {fluid }}=u_{w}
$$

where $u_{w}=\frac{\partial u_{\text {solid }}}{\partial t}$

The above steps are repeated until the solution converges. They are done automatically in COMSOL using the FSI module. Large deformation is formulated through geometric nonlinearity and the ALE method.

\section{Boundary condition}

To achieve the two objectives stated in the Introduction section, different boundary condition settings were applied. For all simulations, the undeformed stress of the myocardium is set to be $3000 \mathrm{~Pa}$, with apex and base of the simulated LV fixed in position.

\section{(i) Effect of the inflow cannula insertion depth}

In order to investigate the effect of the inflow cannula insertion depth on the intraventricular flow and pressure fields, two different placements of cannula were simulated: one near to the apex $(0.35 \mathrm{~cm}$ from the apex $)$ while another was inserted half way into the ventricle $(4.15 \mathrm{~cm}$ from the apex). A sinusoidal velocity profile with a period of $1 \mathrm{~s}$ and a peak magnitude of $0.15 \mathrm{~m} / \mathrm{s}$ was applied at the inlet to simulate repeated diastolic inflow pulses (Fig. 2(a)). A pressure of $0 \mathrm{mmHg}$ was applied at the outlet (the end of cannula).

\section{(ii) Myocardial suction phenomena}

To simulate the myocardial suction phenomena, the boundary condition at the outlet was changed from an inlet pressure of $0 \mathrm{mmHg}$ to a linearly increasing velocity, as shown in Fig.2 (b). On the other hand, the inlet velocity was set to 0 to stop the incoming flow. During this simulation, the cannula tip was inserted half way into the ventricle.

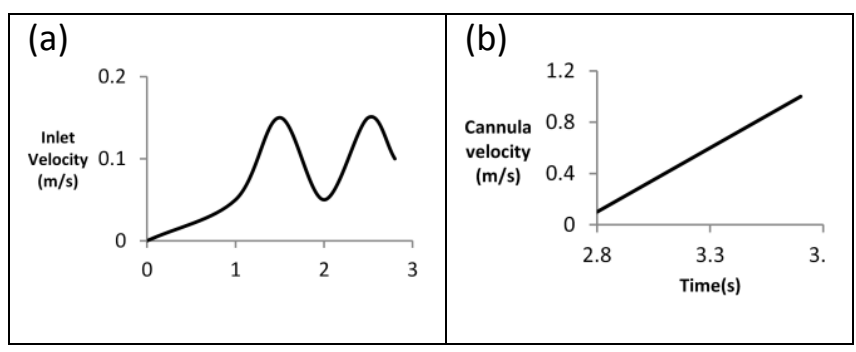

Fig. 2: (a) Sinusoidal velocity profile was applied at the inlet to simulate repeated diastolic inflow pulses. (b) A linearly increasing outflow velocity profile was applied at the end of the cannula to simulate suction. 


\section{RESULTS}

Fig. 3 shows the evolution of the intraventricular vorticity pattern when the inflow cannula was inserted near the apex. A small vortex was formed near the inlet at the early filling phase $(\mathrm{t}=1 \mathrm{~s})$, which gradually rolls up and grows into a vortex ring $(\mathrm{t}=1.8 \mathrm{~s})$. Boundary layer was formed at the basal wall region due to the interaction between the vortex ring and the ventricular wall. During the acceleration phase of the second incoming flow $(\mathrm{t}=2.3 \mathrm{~s})$, it can be observed that the primary vortex ring has detached from the ventricular wall and reached the apex of the ventricle. A second vortex was formed near the inlet due to this incoming flow. During the deceleration phase $(t=2.8 \mathrm{~s})$, the vortex near the inlet of the ventricle has grown into a vortex ring, while the primary vortex has reached the tip of the cannula with a smaller core area as compared to before.

When the cannula was inserted half way into the ventricle (Fig. 4), similar results were obtained at the early filling phase $(\mathrm{t}=1 \mathrm{~s})$, where a small vortex was formed near the inlet. At $\mathrm{t}=$ $1.8 \mathrm{~s}$, the primary vortex ring has reached the cannula tip, and interacts with the boundary layer at the cannula tip to form a large recirculation flow region. During the second incoming flow $(\mathrm{t}=2.3 \mathrm{~s})$, a second vortex was formed near the inlet, while the primary vortex has detached from the cannula tip. The second vortex then grew in size and formed a vortex ring near the cannula tip. Secondary vortices with smaller core area were also observed between the cannula and the ventricular wall.

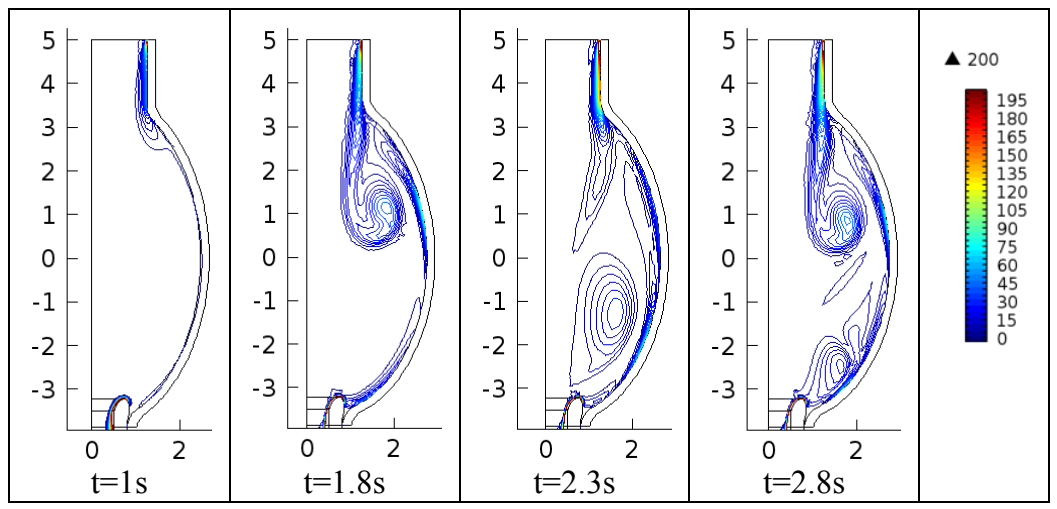

Fig. 3: Instantaneous vorticity pattern when the cannula was inserted near the apex $(0.375 \mathrm{~cm}$ from the apex).

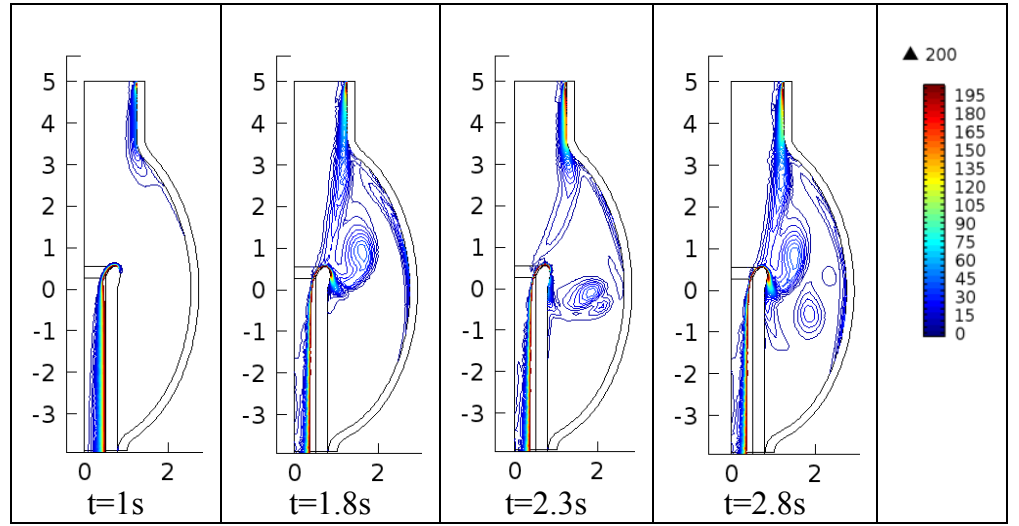

Fig. 4: Instantaneous vorticity pattern when the cannula was inserted half way into the ventricle ( $4.15 \mathrm{~cm}$ from the apex).

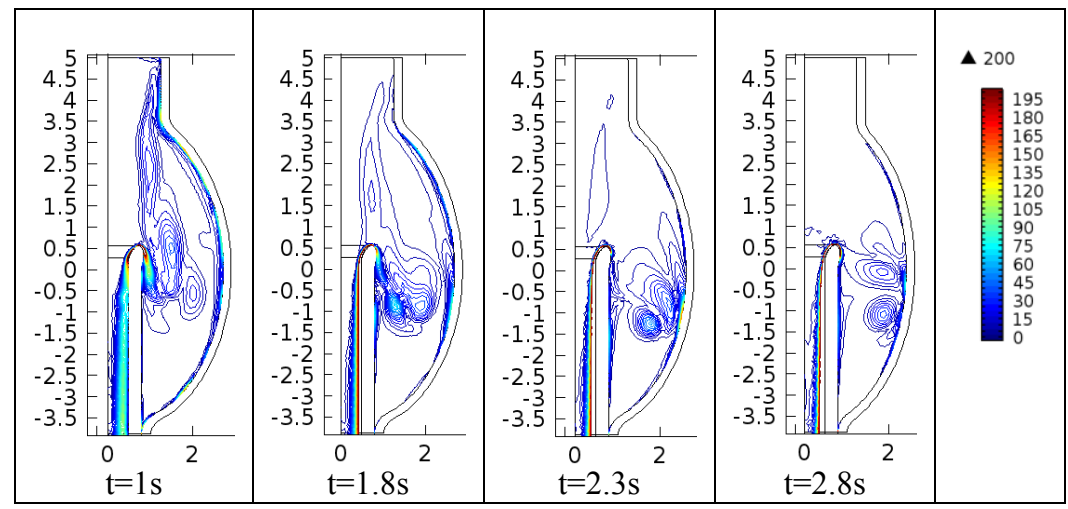

Fig. 5: Instantaneous vorticity pattern during a suction event (with cannula inserted $4.15 \mathrm{~cm}$ from the apex). 
Fig. 5 illustrates the intraventricular vortex dynamics during a suction event. Starting from $t=2.9 \mathrm{~s}$, the outlet velocity was increased linearly, while the inlet velocity was set to zero to stop the incoming flow. Local acceleration of fluid around the cannula tip induced gradual movement of vortices towards the cannula tip. Meanwhile, the wall was also drawn towards the cannula, with the greatest displacement occurring at the region closest to the cannula tip. Secondary vortices can be observed between the cannula and the ventricular wall, where they were constrained by the fluid at the basal region, the cannula wall and the ventricular wall.

\section{DISCUSSION}

Improper placement of the cannula has been associated with an increasing risk of thrombus formation and myocardial suction events [5, 11]. Schmid et al. [12] found a lower rate of thromboembolic complications and a lower incidence of cerebrovascular adverse events in patients with a long inflow cannula. Our simulation results showed a larger vortex core area around the cannula tip when it was placed deeper into the ventricle. As suggested by Fraser et al. [5], increase in the recirculation flow region near the cannula tip increases wash out, thereby reduces the chance of platelet adhesion at the cannula tip.

Although better wash out was observed at the cannula tip with increasing cannula insertion depth, no vortices was found at the apex of the ventricle. This may induce thrombus formation at the apical wall region. This is in contrast to the experimental results reported by Laumen et al. [11], who reported a more distinctive vortex and higher velocity along the ventricular wall when the cannula was inserted deeper into the ventricle. Their results were based on horizontal cut planes viewed from the top of the ventricle, therefore may represent vorticity anywhere between the base and the apex (not solely in the apex). Nevertheless, contraction of the ventricular wall, which was not included in the present study, is likely to flush out the stagnant flow region at the apex region during systole.

\section{CONCLUSION}

We have presented a FSI model of the simulated passive $\mathrm{LV}$, incorporating an inflow cannula, to study the effect of cannula insertion depth and suction events on the intraventricular flow distribution. Our results showed that larger recirculation area was formed at the cannula tip with increasing cannula insertion depth, and this is believed to reduce the risk of occlusion at the cannula tip. Future work includes modelling the contractile activity of the LV to be able to simulate the whole cardiac cycle, as well as validating the results using in vitro and in vivo experiments.

\section{ACKNOWLEDGMENT}

This study was funded by Ministry of Higher Education (MOHE) of Malaysia, grant number UM.C/HIR/MOHE/ENG/14 D000014-16001- and supported by University of Malaya Faculty of Engineering.

\section{REFERENCES}

[1] M. McCormick, D. Nordsletten, D. Kay, and N. Smith, "Modelling left ventricular function under assist device support," International Journal for Numerical Methods in Biomedical Engineering, vol. 27, no. 7, pp. 1073-1095, 2011.

[2] K. H. Fraser, M. E. Taskin, B. P. Griffith, and Z. J. Wu, "The use of computational fluid dynamics in the development of ventricular assist devices," Medical Engineering \&amp; Physics, vol. 33, no. 3, pp. 263-280, 2011.

[3] A. L. Throckmorton, A. Untaroiu, P. E. Allaire, H. G. Wood, G. P. Matherne, D. S. Lim, B. B. Peeler, and D. B. Olsen, "Computational Analysis of an Axial Flow Pediatric Ventricular Assist Device," Artificial Organs, vol. 28, no. 10, pp. 881-891, 2004.

[4] J. Wu, B. E. Paden, H. S. Borovetz, and J. F. Antaki, "Computational Fluid Dynamics Analysis of Blade Tip Clearances on Hemodynamic Performance and Blood Damage in a Centrifugal Ventricular Assist Device," Artificial Organs, vol. 34, no. 5, pp. 402-411, 2010.

[5] K. H. Fraser, T. Zhang, M. E. Taskin, B. P. Griffith, and Z. J. Wu, "Computational fluid dynamics analysis of thrombosis potential in left ventricular assist device drainage cannulae," ASAIO journal (American Society for Artificial Internal Organs : 1992), vol. 56, no. 3, pp. 157-63, 2010.

[6] S. Loerakker, L. G. E. Cox, G. J. F. van Heijst, B. A. J. M. de Mol, and F. N. van de Vosse, "Influence of dilated cardiomyopathy and a left ventricular assist device on vortex dynamics in the left ventricle," Computer Methods in Biomechanics and Biomedical Engineering, vol. 11, no. 6, pp. 649-660, 2008/12/01, 2008.

[7] H. Watanabe, S. Sugiura, H. Kafuku, and T. Hisada, "Multiphysics Simulation of Left Ventricular Filling Dynamics Using Fluid-Structure Interaction Finite Element Method," Biophysical Journal, vol. 87, pp. 2074-2085, 2004.

[8] B. Chahboune, and J. M. Crolet, "Numerical simulation of the blood-wall interaction in the human left ventricle," The European Physical Journal - Applied Physics, vol. 2, no. 03, pp. 291-297, 1998.

[9] Y. Cheng, H. Oertel, and T. Schenkel, "Fluid-Structure Coupled CFD Simulation of the Left Ventricular Flow During Filling Phase," Annals of Biomedical Engineering, vol. 33, no. 5, pp. 567-576, 2005.

[10] S. Dokos, B. H.Smaill, A. A.Young, and I. J.LeGrice, "Shear properties of passive ventricular myocardium," American Journal physiology heart circularion physiology, vol. 283, pp. H2650-H2659, 2002.

[11] M. Laumen, T. Kaufmann, D. Timms, P. Schlanstein, S. Jansen, S. Gregory, K. C. Wong, T. Schmitz-Rode, and U. Steinseifer, "Flow Analysis of Ventricular Assist Device Inflow and Outflow Cannula Positioning Using a Naturally Shaped Ventricle and Aortic Branch," Artificial Organs, vol. 34, no. 10, pp. 798-806, 2010.

[12] C. Schmid, M. Jurmann, D. Birnbaum, T. Colombo, V. Falk, G. Feltrin, A. Garatti, M. Genoni, G. Gerosa, P. Göttel, J. Gummert, R. Halfmann, D. Hammel, E. Hennig, F. Kaufmann, M. Lanfranconi, B. Meyns, F. Mohr, J. Müller, D. Nikolov, K. Rucinskas, H.-H. Scheld, F.-X. Schmid, M. Schneider, V. Sirvydis, R. Tandler, E. Vitali, D. Vlasselaers, M. Weyand, M. Wilhelm, and R. Hetzer, "Influence of Inflow Cannula Length in Axial-flow Pumps on Neurologic Adverse Event Rate: Results From a Multi-center Analysis," The Journal of heart and lung transplantation : the official publication of the International Society for Heart Transplantation, vol. 27, no. 3, pp. 253-260, 2008. 